\title{
Diatom Fingerprinting to Ascertain Death in Drowning Cases
}

\author{
Vandana Vinayak*, Vichar Mishra and Goyal MK
}

Department of Criminology and Forensic Science, H.S. Gour University (Central University) Sagar, Madhya Pradesh, India

\begin{abstract}
Diatoms are unicellular microorganisms which are commonly found in almost all water bodies. Their silica wall plays important tool in forensic diatomology. Diatom test has been widely applied to detect post mortem or antemortem drowning and comparing the diatoms found in biological sample with those found in water sample confirms that death took place, probably in same water medium. However, in skeletonized stage or dismemberment of body parts at different location, diatom of specific kind helps to link the place of drowning. Diatom fingerprinting was thus created studied for 2 years in water bodies of Haryana to differentiate commonly, least commonly found and seasonal diatoms. A total of 111 species and their 55 genera of diatoms were identified. Diatom genera which were commonly found at all the ten sites were Achnanthes, Asterionella, Aulacoseira, Cyclotella, Gomphonema, Navicula, Nitzschia, Synedra and Tabularia. Some of the diatoms were site specific such as, Cymatopleura, Opephora, and Capartogramma and Cyclotella. A new genus of diatom was discovered Eunocymbellarania vandana, which thus make it site specific. Study of seasonal and site specific diatoms at different sites may help in indicating time of drowning along with the location of drowning which would be very helpful for the scientific forensic investigation of drowning cases.
\end{abstract}

Keywords: Diatom fingerprinting; Haryana; Drowning

\section{Introduction}

Every year in India, along with other cause of death "Drowning" plays a major role; it may be Accidental, Suicidal or Homicidal Drowning. Drowning is a type of asphyxial death in which the respiration is inhibited by submersion in a fluid, and it is not necessary whether the fluid is aspirated into the lungs or not.

The diagnosis of drowning for bodies freshly retrieved from water is mainly based on some "drowning signs", such as the presence of fine froth at the mouth or nostrils, petechial haemorrhages, impression of ribs on lungs, oedematous lungs and some other histo-pathological findings. For the decomposed corpses and skeletonised body found in water, however, the diagnosis of drowning is rather difficult because those "drowning signs" were destroyed. Here diatom test stands as the only direct screening test for drowning [1].

The principle behind the "diatom test" is when drowning takes place the diatoms present in medium, enter through ruptured capillaries via penetration into the alveolar system, subsequently to blood stream and further distant organs and other body tissues, it is here diatom test seeks as golden standard test in forensic investigation to detect antemortem or postmortem drowning. The utility of the diatom test for drowning using femoral bone marrow was calculated in a series of 771 cases of drowning that occurred during the summer, late spring and early autumn in Ontario, Canada. The diatom test for drowning was positive in 205 cases $(28 \%)$ of the freshwater drowning cases but was rarely positive if drowning occurred in water from a domestic source that lacked diatoms due to water filtration and processing. Only four cases $(12 \%)$ of domestic drowning had a positive diatom test. The relationship of test outcome with diatom population dynamics is important corroborating evidence for the validity of the test since it is predictable that the diatom content of the water would influence test outcome [2,3]. The postmortem analysis of drowning is a classical problem in the pathology of sudden violent death. From a historical perspective, one of the most important issues in the study of drowning has been the search for a sensitive, specific and easily applicable test for this cause of death. On this basis, the diatom test has emerged as the foremost laboratory procedure for the detection of drowning [3]. 29 cases of drowning were analyzed and emphasize for the usefulness of the diatom-test to find the manner of death and stressed on the collaboration between forensic pathologists and specialist- biologists, who could work together to generate the diatomologic map of the water bodies of that region. In this way, some rare types of diatoms can be identified and located, which can be of great help in pinpointing the actual site of drowning [4].

The presence of diatoms can be established and analyzed both quantitatively and qualitatively through a diatom test. This can lead not only to a more direct determination of the cause of death, but also can help to pinpoint the site of a suspected drowning [5].

Continuous monitoring of water bodies for the percentage bloom of diatom flora and its taxonomy is very essential every year. Since due to climate changes there is variability in diatom flora, which serves as an effective tool to diagnose death due to drowning [6]. Many time body get drifted to some other place either due to water current or otherwise. In such cases if a thorough monitoring of diatom flora of a particular body is recorded, it becomes possible to locate site and season of drowning. For this purpose database of most commonly occurring, site specific, and seasonal occurring diatoms was constituted along with the shape and size resulting into the formation of a fingerprinting system for diatoms.

*Corresponding author: Vandana Vinayak, Department of Criminology and Forensic Science, School of Applied Sciences, Dr. H.S. Gour University (Central University) Sagar, Madhya Pradesh, India, Tel: +91 9179577953; E-mail: kapilvinayak@gmail.com

Received September 18, 2013; Accepted November 20, 2013; Published November 23, 2013

Citation: Vinayak V, Mishra V, Goyal MK (2013) Diatom Fingerprinting to Ascertain Death in Drowning Cases. J Forensic Res 4: 207. doi:10.4172/2157-7145.1000207

Copyright: $\odot 2013$ Vinayak V, et al. This is an open-access article distributed under the terms of the Creative Commons Attribution License, which permits unrestricted use, distribution, and reproduction in any medium, provided the original author and source are credited. 


\section{Materials and Methods}

\section{Sampling of water from different water bodies of Haryana}

Two liter water sample was collected in a sterilized plastic bottle from ten different water bodies (Table 1) of Haryana (Figure 1) every month for a period of two years (2008-2010). At the time of sampling, temperature, $\mathrm{pH}$ and conductivity was measured using $\mathrm{pH}$ conductivity probe (Swastika ltd., India). Site of sample collection was located by landmarks such as tree, stone or simply a flag.

The water sample was brought in the laboratory and was stored in a cool dark place, to it was added $1 \mathrm{ml}$ of $0.2 \%$ formalin to restrict further division and growth. Slide was prepared by Lugol's iodine method given by Witzel and Likens [7] and under Compound microscope attached with camera (Leica DM 2500) at 100x (Oil immersion) slide was observed and there morphological and morphometric studies were done using LAS EZ software provided with the microscope.

\section{Morphological identification}

Acquired images of diatoms were identified using identification keys of Hans [2,8-19].

Distribution pattern and variation of different types of diatoms in all the three types of water bodies (canal, lake and pond) was characterized as follows:

- Commonly occurring or Dominating diatoms

- Least occurring or Site specific diatoms

- Seasonal or Rarely occurring diatoms

Commonly occurring (dominating) diatoms, are defined as the diatoms found in large percentage than others, and mostly they were present in all seasons at all the sites. Least occurring diatoms were those which were generally present in all the seasons but there count was less than the dominating ones thus are site specific. Rarely occurring diatoms one those which were observed only in particular season thus also known as seasonal diatom (Plate 1).

\section{Results and Discussion}

Diatoms are sensitive to changes in $\mathrm{pH}$, conductivity, temperature, and thus variation in the water quality enables them to function as environmental, ecological and forensic indicators. For two consecutive years study of Diatoms from different water bodies of Haryana from ten water bodies (Table 1) was done and a total 111 diatom taxa were studied [6]. The most commonly found diatom at all the ten sites were Achnanthes, Asterionella, Aulacoseira, Cyclotella, Gomphonema, Navicula, Nitzschia, Synedra and Tabularia.

\section{Canal sites (H1, H2 and H6)}

The commonly occurring diatoms at the canal water bodies were Asterionella, Cocconeis, Cymbella, Navicula. Site specific diatom at $\mathrm{H1}$ was Cymatopleura, Opephora, and Capartogramma at H2 Catenula, Didymosphenia, Stenopterobia and at H6 it was Denticula, Luticola, and Staurosira. Seasonal diatom at H1 was Opephora, at H2 was Catenula, and at $\mathrm{H} 6$ it was Luticola.

\section{Lake sites (H4, H5 and H9)}

The commonly occurring diatoms at lake water bodies were Asterionella, Cyclotella, and Cymbella where as the site specific at H4 was Brachysira and Thalassiosira, at H5 it was Licmophora and Rhopalodia, and at H9 it was Denticula and Reimeria. Seasonal diatom at $\mathrm{H} 4$ was Actinocyclus, at $\mathrm{H} 5$ was Eunocymbellarania [6] and at $\mathrm{H} 9$ it was Epithemia.

\section{Pond sites (H3, H7, H8 and H10)}

The commonly occurring diatom genera at pondwater bodies were Cyclotella, Navicula and Fragilaria. Site specific at H3 were Coscinodiscus, Diploneis, and Eunotia, at H7 Rhopalodia at H8 it was Cyclotella and at $\mathrm{H} 10$ it was Coscinodiscus. Seasonal diatom at $\mathrm{H} 3$ was Eunotia, at $\mathrm{H} 7$ was Eunocymbellarania [6] at $\mathrm{H} 8$ was Coscinodiscus and at $\mathrm{H} 10$ the seasonal diatom was Gomphonema. Figure 2 shows few commonly occurring diatoms, Figure 3 shows some least occurring, and Figure 4 shows some seasonal diatoms). Diatom fingerprinting (Plate 1) of different diatom taxas at different water body would thus help in generating a database to link scene of actual drowning and thus would be of great aid in criminal justice delivery system.

\begin{tabular}{|c|c|c|c|c|}
\hline Station Code & Water body & Source & Geographical area type & Location \\
\hline $\mathrm{H}-1$ & Canal & Western Eastern Yamuna Canal, Panipat & Plain & $\begin{array}{l}29^{\circ} 21^{\prime} 41.44^{\prime \prime} \mathrm{N} \\
76^{\circ} 57^{\prime} 05.58^{\prime \prime} \mathrm{E}\end{array}$ \\
\hline $\mathrm{H}-2$ & Canal & Western Eastern Yamuna Canal, Karnal & Plain & $\begin{array}{l}29^{\circ} 37^{\prime} 18.29 " \mathrm{~N} \\
76^{\circ} 58^{\prime} 56.16^{\prime \prime} \mathrm{E}\end{array}$ \\
\hline $\mathrm{H}-3$ & Pond & Muncipal park, Ambala & Plain & $\begin{array}{l}30^{\circ} 22^{\prime} 38.78 " \mathrm{~N} \\
76^{\circ} 46^{\prime} 19.95^{\prime \prime} \mathrm{E}\end{array}$ \\
\hline $\mathrm{H}-4$ & Lake & Bhramsarover Lake, Kurukshetra & Plain & $\begin{array}{l}29^{\circ} 57^{\prime} 43.10^{\prime \prime} \mathrm{N} \\
76^{\circ} 49^{\prime} 40.32^{\prime \prime} \mathrm{E}\end{array}$ \\
\hline $\mathrm{H}-5$ & Lake & Rani Taalab Lake, Jind & Plain & $\begin{array}{l}29^{\circ} 18^{\prime} 53.06^{\prime \prime} \mathrm{N} \\
76^{\circ} 19^{\prime} 03.00^{\prime \prime} \mathrm{E}\end{array}$ \\
\hline $\mathrm{H}-6$ & Canal & Bhakra Canal, Hisar & Plain & $\begin{array}{l}29^{\circ} 07^{\prime} 51.86^{\prime \prime} \mathrm{N} \\
75^{\circ} 42^{\prime} 33.41^{\prime \prime} \mathrm{E}\end{array}$ \\
\hline $\mathrm{H}-7$ & Pond & Ghaseda village, Mewat & Foot hills & $\begin{array}{l}28^{\circ} 07^{\prime} 57.26^{\prime \prime} \mathrm{N} \\
77^{\circ} 03^{\prime} 53.41^{\prime \prime} \mathrm{E}\end{array}$ \\
\hline $\mathrm{H}-8$ & Pond & Baghot village, Mahendergarh & Foot hills & $\begin{array}{l}28^{\circ} 25^{\prime} 31.72 " \mathrm{~N} \\
76^{\circ} 15^{\prime} 55.69^{\prime \prime} \mathrm{E}\end{array}$ \\
\hline $\mathrm{H}-9$ & Lake & Tilyar Lake, Rohtak & Plain & $\begin{array}{l}28^{\circ} 52^{\prime} 52.60^{\prime \prime} \mathrm{N} \\
76^{\circ} 38^{\prime} 16.20^{\prime \prime} \mathrm{E}\end{array}$ \\
\hline $\mathrm{H}-10$ & Pond & Baund village, Bhiwani & Plain & $\begin{array}{l}28^{\circ} 46^{\prime} 44.64^{\prime \prime} \mathrm{N} \\
76^{\circ} 20^{\prime} 09.59^{\prime \prime} \mathrm{E}\end{array}$ \\
\hline
\end{tabular}

Table 1: Selected water stations for sampling from Haryana (India). 
Citation: Vinayak V, Mishra V, Goyal MK (2013) Diatom Fingerprinting to Ascertain Death in Drowning Cases. J Forensic Res 4: 207. doi:10.4172/21577145.1000207

Page 3 of 6

\begin{tabular}{|c|c|c|c|c|c|c|c|c|c|c|c|c|c|c|c|c|}
\hline \multirow{2}{*}{\multicolumn{2}{|c|}{ Sr. No. }} & \multirow{2}{*}{ Diatom genera } & \multicolumn{10}{|c|}{ Station code } & \multirow{2}{*}{ Order } & \multirow{2}{*}{ Shape } & \multicolumn{2}{|c|}{ Size $(\mu \mathrm{m})$} \\
\hline & & & H1 & $\mathrm{H} 2$ & $\mathrm{H} 3$ & $\mathrm{H} 4$ & H5 & H6 & H7 & H8 & H9 & H10 & & & Length & Width \\
\hline 1. & 1 & Achnanthes & + & + & & + & + & + & & + & + & + & Pennale & Elliptical & $8-16$ & $4-6$ \\
\hline 2. & 2 & Actinocydus & & & & & & & & & & & Centrale & Circular & $36-40$ & - \\
\hline 3. & 3 & Amphora & 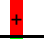 & & & & & & & & & & Pennale & Elliptical & $75-80$ & $15-20$ \\
\hline 4. & 4 & Asterionella & + & + & + & + & + & + & + & & + & + & Pennale & Elongated & $80-300$ & $5-8$ \\
\hline 5. & 5 & Aulacoseira & + & + & & + & + & + & + & & + & + & Centrale & Circular & $45-50$ & - \\
\hline 6. & 6 & Brachysira & & & & & & & & & & & Pennale & Elliptical & $20-48$ & $12-20$ \\
\hline 7. & 7 & Caloneis & & & & & & & & & & & Pennale & Elliptical & $22-50$ & $10-14$ \\
\hline 8. & 8 & Capartogramma & & & & & & & & & & & Pennale & Elliptical & $18-45$ & $12-18$ \\
\hline 9. & 9 & Catenula & & & & & & & & & & & Pennale & Elliptical & $10-15$ & $2-5$ \\
\hline 10. & 10 & Craticula & & + & + & & & + & + & + & + & + & Pennale & Elliptical & $44-200$ & $25-75$ \\
\hline 11. & 11 & Cocconeis & + & + & + & + & & + & & & & & Pennale & Oval & $35-45$ & $16-17$ \\
\hline 12. & 12 & Coscinodiscus & & & & & & & & & & & Centrale & Circular & $36-120$ & - \\
\hline 13. & 13 & Cyclotella & + & + & + & + & + & + & + & & + & + & Centrale & Circular & $6-40$ & - \\
\hline 14. & 14 & Cymatopleura & . & & & & & & & & & & Pennale & Shoe & $80-300$ & $10-45$ \\
\hline 15. & 15 & Cymbella & + & + & + & + & + & + & + & & + & & Pennale & Elliptical & $20-200$ & $5-50$ \\
\hline 16. & 16 & Denticula & & & & & & & & & & & Pennale & Elliptical & $11-14$ & $3-5$ \\
\hline 17. & 17 & Diatoma & + & + & + & & & + & + & & & & Pennale & Elliptical & $18-60$ & $8-18$ \\
\hline 18. & 18 & Didymosphenia & & & & & & & & & & & Pennale & Elliptical & $80-150$ & $30-50$ \\
\hline 19. & 19 & Diploneis & + & + & & & & + & & & & & Pennale & Elliptical & $10-40$ & $6-10$ \\
\hline 20. & 20 & Entomoneis & + & & & & & + & & & & & Pennale & Sigmoid & $95-120$ & $40-60$ \\
\hline 21. & 21 & Epithemia & + & & + & & & & & & & & Pennale & 'Elliptical & $40-50$ & $8-10$ \\
\hline 22. & 22 & Encyonema & + & + & & & & + & + & & + & + & Pennale & Elliptical & $20-60$ & $4-35$ \\
\hline 23. & 23 & Eunocymbellarania & & & & & & & + & & + & & pennale & Elliptical & $20-150$ & $5-25$ \\
\hline 24. & 24 & Eunotia & + & + & + & & & + & & & + & & Pennale & Elliptical & $22-28$ & $6-8$ \\
\hline 25. & 25 & Fragilaria & + & & + & & + & + & + & + & + & + & Pennale & Elliptical & $95-119$ & $6-7.2$ \\
\hline 26. & 26 & Frustulia & + & & & + & & + & & & & + & Pennale & Elliptical & $60-85$ & $10-18$ \\
\hline 27. & 27 & Gomphoneis & & + & + & & + & & & & & + & Pennale & Elliptical & $20-130$ & $12-20$ \\
\hline 28. & 28 & Gomphonema & + & + & + & + & + & + & + & + & + & + & Pennale & Elliptical & $74-90$ & $16-20$ \\
\hline 29. & 29 & Gyrosigma & + & + & 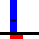 & + & & + & & & + & + & Pennale & Sigmoid & $150-240$ & $26-30$ \\
\hline 30. & 30 & Gomphocymbella & & & + & & & & & & & & Pennale & Elliptical & $18-30$ & $5-8$ \\
\hline 31. & 31 & Hantzschia & & + & + & + & + & + & & + & & & Pennale & Elongate & $80-120$ & $18-22$ \\
\hline 32. & 32 & Licmophora & & & & & & & & & & & Pennale & Elliptical & $45-60$ & $5-7$ \\
\hline 33. & 33 & Luticola & & & & & & & & & & & Pennale & Elliptical & $15-20$ & $5-10$ \\
\hline 34. & 34 & Melosira & + & & + & + & + & & + & & + & + & Centrale & Circular & $23-25$ & - \\
\hline 35. & 35 & Navicula & + & + & + & + & + & + & + & + & + & + & Pennale & Elliptical & $10-60$ & $5-16$ \\
\hline 36. & 36 & Neidium & + & + & 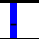 & + & & & & + & 1 & + & Pennale & Elliptical & $40-50$ & $12-20$ \\
\hline 37. & 37 & Nitzschia & + & + & + & & + & + & + & + & + & + & Pennale & Elliptical & $25-115$ & $5-10$ \\
\hline 38. & 38 & Opephora & + & & & & & & & & & & Pennale & Elliptical & $30-35$ & $5-7$ \\
\hline 39. & 39 & Pinnularia & + & + & + & + & + & + & & & & + & Pennale & Elliptical & $20-250$ & $8-18$ \\
\hline 40. & 40 & Placoneis & + & + & + & + & + & + & & & & + & Pennale & Elliptical & $20-30$ & $10-12$ \\
\hline 41. & 41 & Pseudostaurosira & + & + & + & & + & + & + & + & + & + & Pennale & Elliptical & $12-22$ & $6-8$ \\
\hline 42. & 42 & Reimeria & + & + & T & & & & & & + & 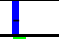 & Pennale & Elliptical & $8-12$ & $4-6$ \\
\hline 43. & 43 & Rhoicosphenia & + & + & + & + & + & + & & & + & + & Pennale & Elliptical & $16-46$ & $8-10$ \\
\hline 44. & 44 & Rhopalodia & & T & + & & + & & + & & + & & Pennale & Elliptical & $18-150$ & $4-8$ \\
\hline 45. & 45 & Sellaphora & + & + & T & & + & + & + & & + & + & Pennale & Elliptical & $18-45$ & $4-10$ \\
\hline 46. & 46 & Stauroneis & & + & + & + & & & & & & & Pennale & Elliptical & $40-75$ & $14-20$ \\
\hline 47. & 47 & Staurosira & & + & 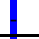 & & & & & & & & Pennale & Elliptical & $7-22.5$ & $5-7$ \\
\hline 48. & 48 & Stenopterobia & & + & & & & & & & & & Pennale & Sigmoid & $80-100$ & $8-16$ \\
\hline 49. & 49 & Staurosirella & & 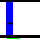 & & & & & & & & & Pennale & Elliptical & $5-10$ & $2-5$ \\
\hline 50. & 50 & Sunirella & + & + & + & & + & + & + & & + & + & Pennale & Elliptical & $40-70$ & $30-40$ \\
\hline 51. & 51 & Synedra & + & + & + & + & + & + & + & & + & + & Pennale & Rod & $93-150$ & $7-9$ \\
\hline 52. & 52 & Tabularia & + & + & + & + & + & + & + & + & + & + & Pennale & Elliptical & $20-120$ & $4-6$ \\
\hline 53. & 53 & Tryblionella & + & + & & 1 & & + & & + & & + & Pennale & Elliptical & $18-40$ & $5-10$ \\
\hline 54. & 54 & Thalassionema & + & + & & + & + & & & & & & Pennale & Elongate & $20-66$ & $2-4$ \\
\hline 55. & 55 & Thalassiosira & & & & & & & & & & & Centrale & Circular & $15-20$ & - \\
\hline
\end{tabular}

Symbols used:

- $\quad(\mathrm{H} 1$ to $\mathrm{H} 10)$ : Sites of water sample collection

- (\#): Present.

- (1): Absent.

- (+): Restricted (rarely occurring and seasonal)

Plate 1: Diatom fingerprinting. 


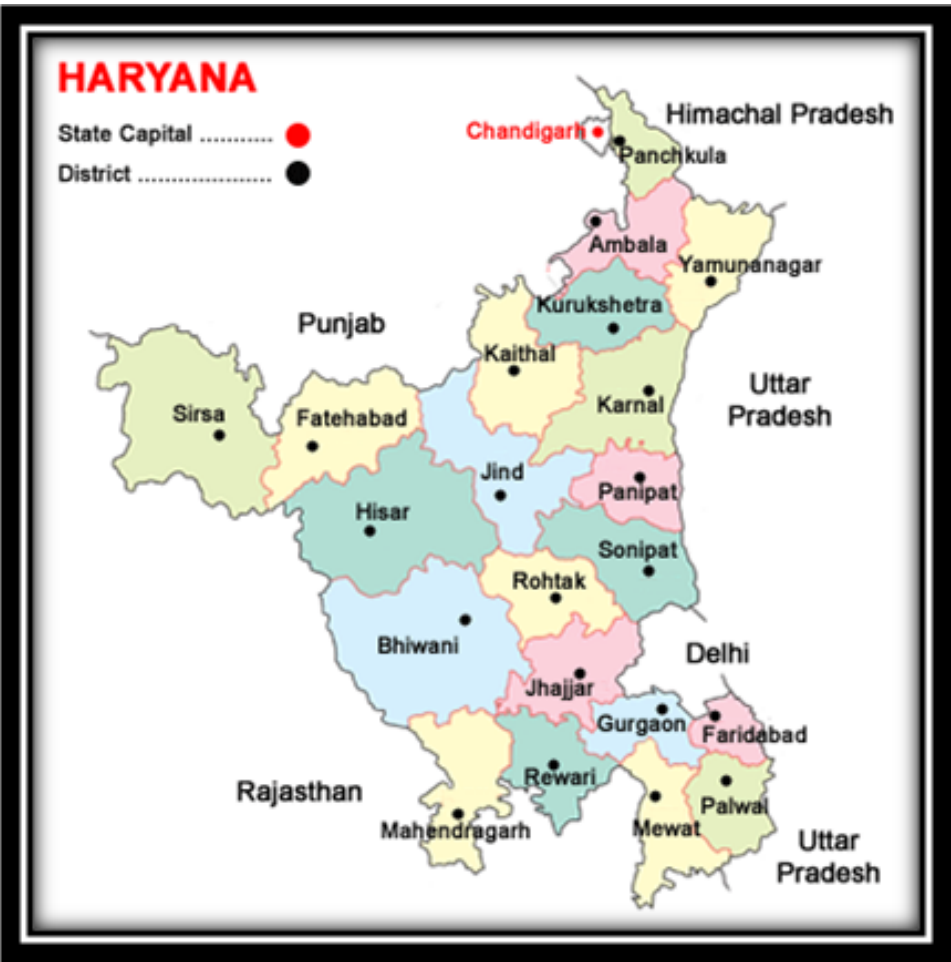

Figure 1: Geographical Map of Haryana.

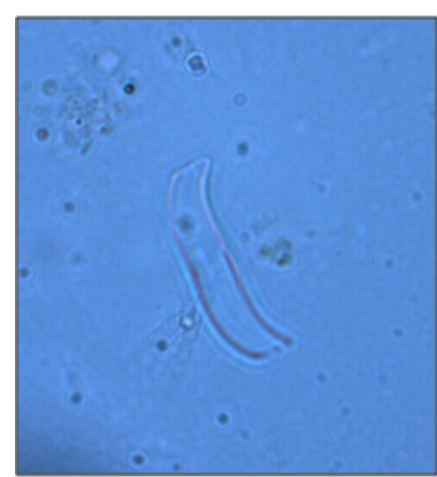

Achnanthes minutissima

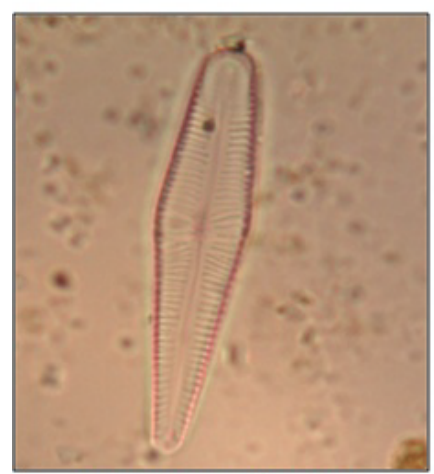

Gomphonema clavatum

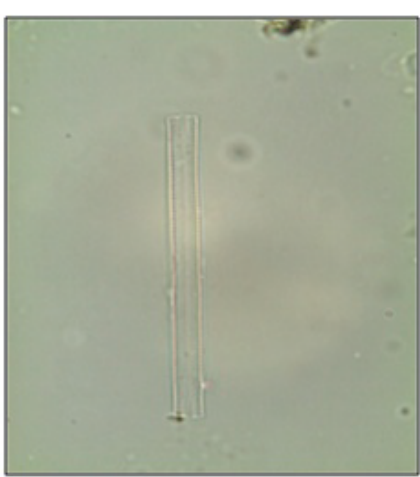

Asterionella formosa

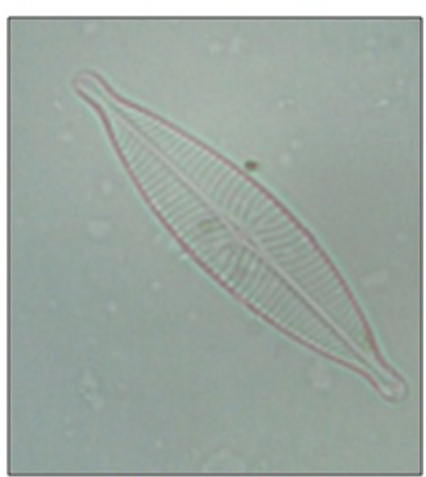

Navicula capitoradiata

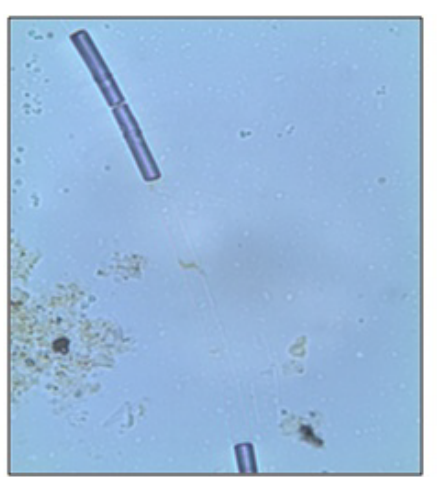

Melosira varians

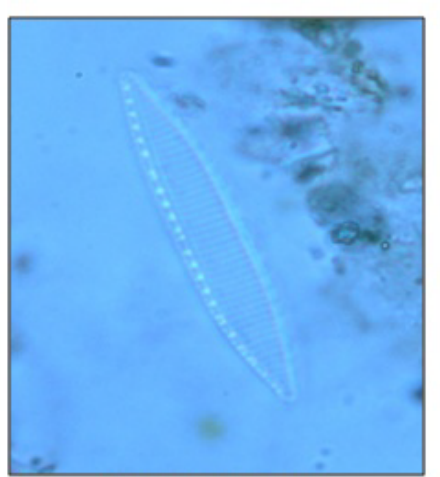

Nitzschia angustata

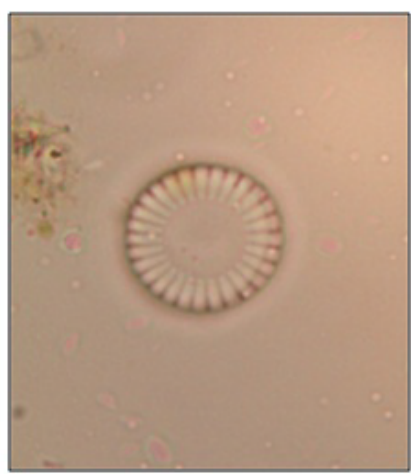

Cyclotella meneghiniana

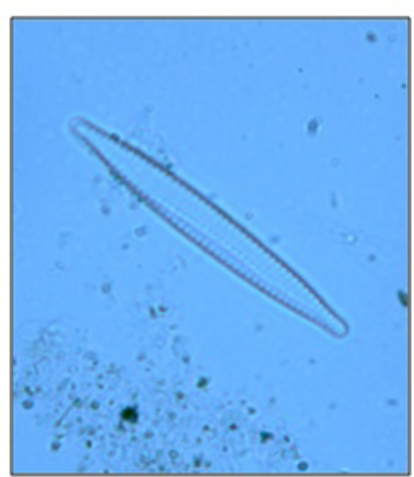

Tabularia fasciculata

Figure 2: Few Commonly occurring diatoms. 
Citation: Vinayak V, Mishra V, Goyal MK (2013) Diatom Fingerprinting to Ascertain Death in Drowning Cases. J Forensic Res 4: 207. doi:10.4172/21577145.1000207

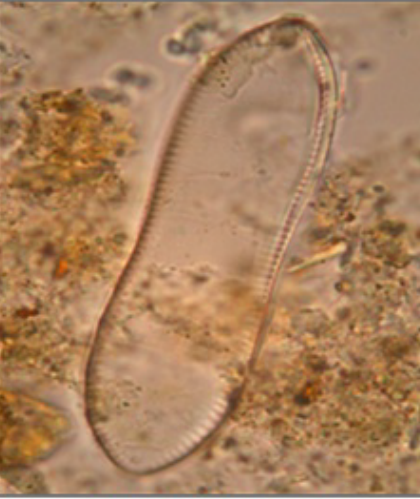

Cymatopleura solea

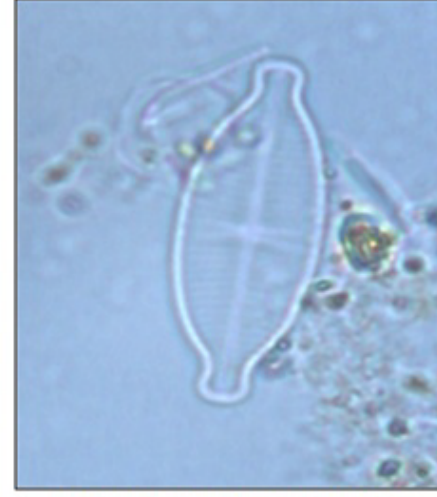

Capartogramma crucicula

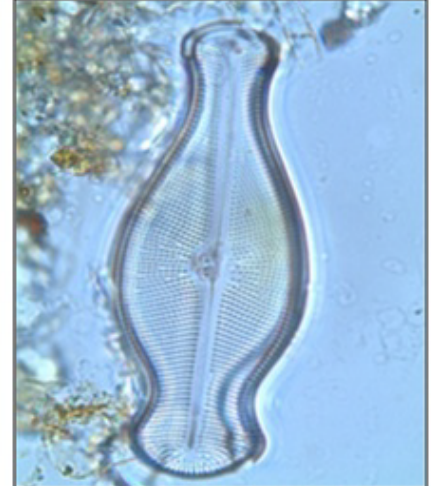

Didymosphenia germinata

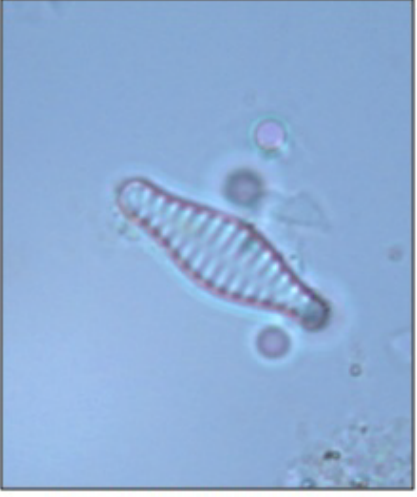

Staurosirella leptostauron

Figure 3: Few Least occuring diatoms.

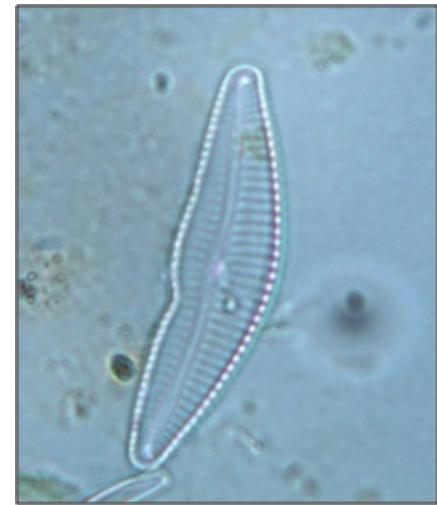

Eunocymbellarania vandana

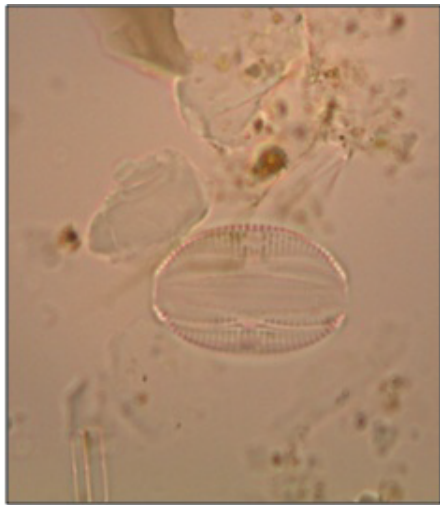

Amphora ovalis

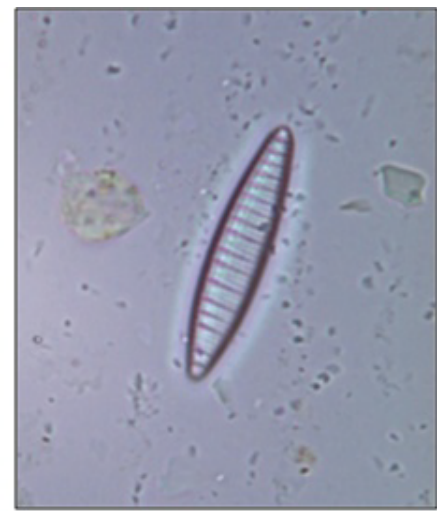

Denticula tenuis

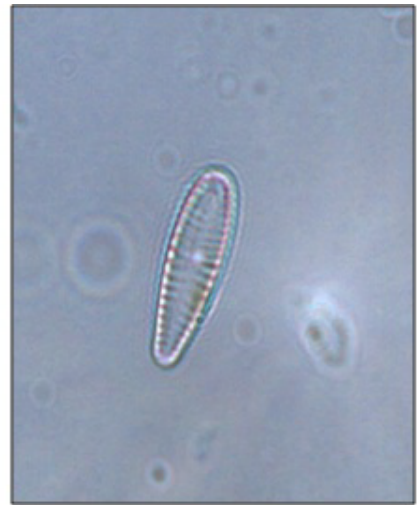

Opephora pacifica

Figure 4: Few Seasonal diatoms.

\section{Acknowledgements}

Author is thankful to Department of Science and Technology, Haryana State Council for science and Technology, India for sanctioning the funds.

\section{References}

1. He F, Huang D, Liu L, Shu X, Yin H, et al. (2008) A novel PCR-DGGE-based method for identifying plankton 16S rDNA for the diagnosis of drowning. Forensic Sci Int 176: 152-156.

2. Pollanen MS, Cheung C, Chiasson DA (1997) The diagnostic value of the diatom test for drowning 1. Utility: a retrospective analysis of 771 cases of drowning in Ontario, Canada. J Forensic Sci 42: 281-285.

3. Pollanen MS (1998) Forensic Diatomology and Drowning. Elsevier, Amsterdam, The Netherlands 1-157.

4. Natasha D, Aleksej D (2005) Differential diagnostic elements in the determination of drowning. Rom J Leg Med 13: 22-30.

5. Ludes B, Coste M, North N, Doray S, Tracqui A et al. (1999) Diatom Analysis in Victim's Tissues as an Indicator of the site of Drowning. International Journal of Legal Medicine 112: 163-166.

6. Vinayak V, Goyal MK, Mishra V (2012) New genus and species of diatom endemic in lake Rani of Haryana, India. Open Journal of Modern Hydrology 2: 99-105.

7. Wetzel RG, Likens GE (1991) Limnological Analysis, 2ndedn., Springer, Berlin Heidelberg, New York.

8. du Buf JMH, Bayer MM (eds.) (2002) Automatic Diatom Identification, Series in
Machine Perception and Artificial Intelligence, World Scientific Publishing Co., Singapore. 51: 289-298.

9. Round FE, Crawford RM, Mann DG (1990) The Diatoms: Biology and Morphology of the genera. Cambridge University Press, Cambridge, UK, 1-747.

10. Patrick R, Reimer CW (1966) The Diatoms of the United States, Exclusive of Alaska and Hawaii, Volume 1, Fragilariaceae, Eunotiaceae, Achnanthaceae, Naviculaceae. Academy of Natural Sciences of Philadelphia Monograph 13 688.

11. Patrick R, Reimer CW (1975)The Diatoms of the United States, Exclusive of Alaska and Hawaii, Volume 2, Part 1 - Entomoneidaceae, Cymbellaceae, Gomphonemaceae, Epithemaceae. Academy of Natural Sciences of Philadelphia Monograph 13: 213.

12. Krammer K, Lange-Bertalot H (1986) Bacillariophyceae 1. Teil. Naviculaceae. Subwasserflora von Mitteleuropa. G. Fisher, Stuttgart. Band 2/1: 1-876.

13. Krammer K, Lange-Bertalot H (1988) Bacillariophyceae. 2. Teil. Bacillariaceae Epithemiaceae, Surirellaceae. Subwasserflora von Mitteleuropa. G. Fisher, Stuttgart. Band 2/2: 1-596.

14. Krammer K Lange-Bertalot $H$ (1991a) Bacillariophyceae. 3. Teil. Centrales, Fragilariaceae, Eunotiaceae. Subwasserflora von Mitteleuropa. G. Fisher, Stuttgart. Band 2/3: 1-576.

15. Krammer K, Lange-Bertalot H (1991b) Bacillariophyceae. 4. Teil. Achnanthaceae. Subwasserflora von Mitteleuropa. G. Fisher, Stuttgart, Band 2/4: 1-437.

16. Krammer K, Lange-Beratlot H (2000) Bacillariophyceae. 3. Teil. Centrales, 
Citation: Vinayak V, Mishra V, Goyal MK (2013) Diatom Fingerprinting to Ascertain Death in Drowning Cases. J Forensic Res 4: 207. doi:10.4172/21577145.1000207

Page 6 of 6

Fragilariaceae, Eunotiaceae. Subwasserflora von Mitteleuropa. Spektrum Akademischer Verlag, Heidelberg, Band 2/3: 599.

17. Simonsen R (1987) Atlas and catalogue of the diatom types of Friedrich Hustedt. J. Cramer, Berlin: 1-772.
18. Gandhi HP (1959) Freshwater diatoms from Sagar in Mysore state. J Indian Bot Soc 38: 305-331.

19. Vinayak V (2012) Diatom atlas of Fresh water bodies from Haryana (Diatom Atlas). LAP, Lambert academic publishing: 1-105. 